\title{
The HAWC experiment and its sensitivity to gamma-ray bursts
}

\section{Zaborov for the HAWC collaboration}

Department of Physics, Pennsylvania State University, 104 Davey Laboratory, University Park, PA 16802, USA

E-mail: dnz2@psu. edu

\begin{abstract}
The High Altitude Water Cherenkov Observatory (HAWC) is an air shower array currently under construction in Mexico at an altitude of 4100 m. HAWC will consist of 300 large water tanks covering an area of about 22000 square meters and instrumented with 4 photomultipliers each. The experimental design allows for highly efficient detection of photon-induced air showers in the $\mathrm{TeV}$ and sub-TeV range and gamma-hadron separation. We show that HAWC has a realistic opportunity to observe the high-energy power law components of GRBs that extend at least up to $30 \mathrm{GeV}$. In particular, HAWC will be capable of observing events similar to GRB 090510 and GRB 090902B. The observations (or non-observations) of GRBs by HAWC will provide information on the high-energy spectra of GRBs. An engineering array consisting of 6 water tanks was operated at the HAWC site since September 2011, collecting over 3 months of data. An upper limit on high energy emission from GRB 111016B is derived from these data.
\end{abstract}

Gamma-Ray Bursts 2012 Conference - GRB2012,

May 07-11, 2012

Munich, Germany 


\section{Introduction}

Recent observations by Fermi LAT suggest that the high-energy emission of some GRBs extends at least to $30 \mathrm{GeV}$. In particular, a hard power law component was detected in the spectra of the long GRB 090902B [1] and the short GRB 090510 [2]. The highest energy photon recorded from GRB 090902B was $33 \mathrm{GeV}$, or $94 \mathrm{GeV}$ corrected for redshift. The high energy component, not described by the Band function [3], is currently a challenge to GRB models. During the propagation of gamma rays through the interstellar medium, an attenuation due to interactions with the extra-galactic background light (EBL) is expected. Consequently, a spectral energy cutoff can be a probe for the EBL density or source properties (e.g. bulk Lorentz factor). The extension of the observations to higher energies requires a detector with a large effective area. This can be provided by Imaging Atmospheric Cherenkov Telescopes (IACTs) and Extensive Air Shower (EAS) arrays. The chances of prompt observations of a GRB by IACTs are, however, limited by the small duty cycle $(\approx 10 \%)$ and restricted field of view ( $5^{\circ}$ in diameter or less). The EAS arrays provide a large field of view $(\approx 2 \mathrm{sr})$ and near $100 \%$ duty cycle. An energy threshold $<100 \mathrm{GeV}$ can be achieved by placing the array at a high altitude (closer to the air shower maximum). So far the searches for GRBs with EAS arrays have not yielded positive detections due to insufficient sensitivity of the existing arrays (although tentative detections have been claimed by several experiments, e.g. Milagrito [4]).

\section{The HAWC observatory}

The High Altitude Water Cherenkov (HAWC) observatory is a very high-energy $(\sim \mathrm{TeV})$ gammaray detector currently under construction near the peak of Volcán Sierra Negra, Mexico. HAWC is located at $4100 \mathrm{~m}$ altitude, $\mathrm{N} 18^{\circ} 59^{\prime} 48^{\prime \prime}$, W $97^{\circ} 18^{\prime} 34^{\prime \prime}$. When completed in 2014, HAWC will consist of 300 water tanks of $7.3 \mathrm{~m}$ diameter and $4.5 \mathrm{~m}$ depth, covering an area of about $22000 \mathrm{~m}^{2}$. Four large photomultiplier tubes (PMTs) will be located near the bottom of each tank. ${ }^{1}$ The PMTs capture Cherenkov light produced in water by the charged particles that compose an extensive air shower. Hit arrival times are used to reconstruct the incident direction of the shower. The rejection of hadronic showers relies on the shower lateral size and high amplitude pulses produced by muons. The effective area (Fig. 1, left) approaches $\approx 10^{5} \mathrm{~m}^{2}$ at $E>3 \mathrm{TeV}$. An angular resolution of $0.1^{\circ}$ can be achieved at $E>5 \mathrm{TeV}$. The energy threshold in triggered mode is about $30 \mathrm{GeV}$. Scalers, the second data acquisition system (DAQ), will measure PMT counting rates. A sudden increase in counting rates may reveal a GRB. This method provides an energy threshold of a few GeV.

The discovery potential of HAWC is illustrated in Fig. 1, right. This assumes that the GRB search time window and celestial coordinates can be set based on satellite observations. It can be seen that the sensitivity of HAWC is comparable to Fermi LAT's sensitivity above $10 \mathrm{GeV}$, but depends strongly on the spectral cutoff (redshift). HAWC scalers complement the main DAQ, covering short GRBs with soft spectra and high redshifts (cutoffs at $E<100 \mathrm{GeV}$ ). The brightest GRBs detected by Fermi, such as GRB 090510, should be observable with HAWC if the cutoff is above $\approx 100 \mathrm{GeV}$. For a detailed report on HAWC sensitivity to GRBs see [5].

\footnotetext{
${ }^{1}$ The effective area and sensitivity estimates presented below correspond to simulations of only three PMTs per tank and are therefore conservative.
} 

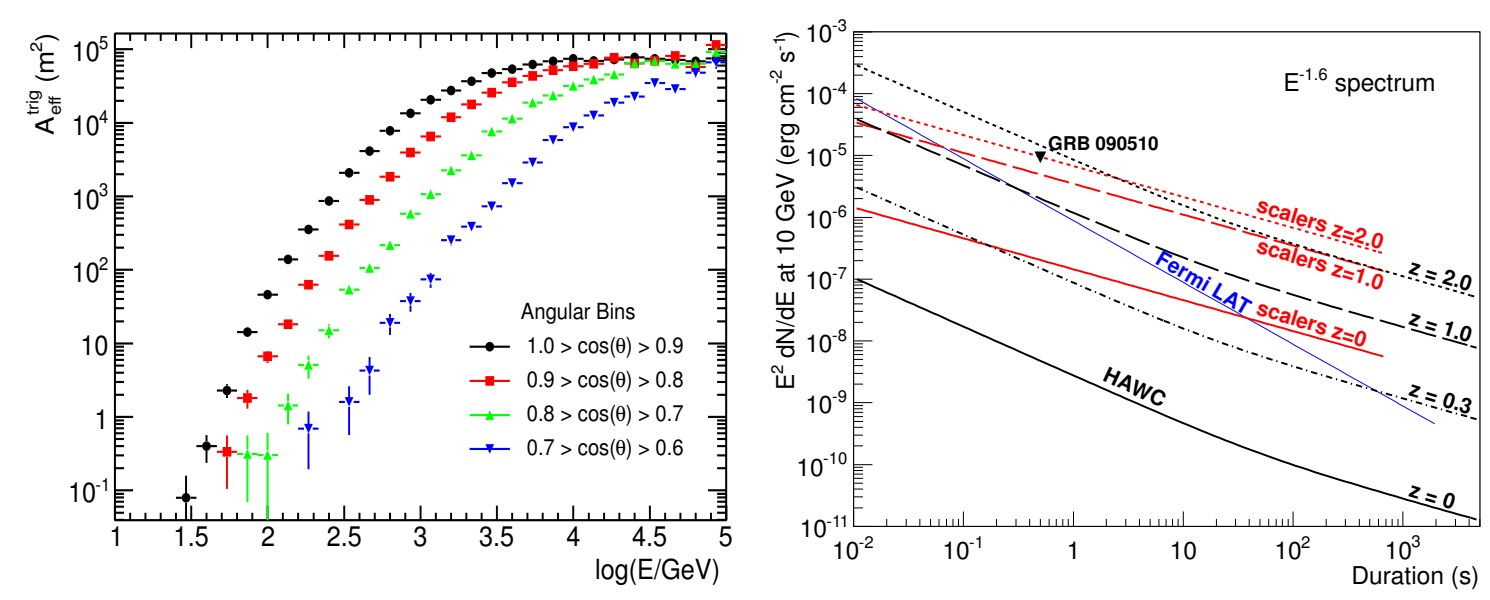

Figure 1: Left: Effective area of HAWC for photon-induced showers. A trigger threshold of 70 PMT hits is assumed. Showers reconstructed with $>0.8^{\circ}$ error are excluded. No gamma-hadron separation cut is applied. Right: Sensitivity ( $5 \sigma$ discovery potential) of HAWC using the main DAQ and the scaler DAQ as a function of burst duration. The source zenith angle is set to $20^{\circ}$. The Gilmore model of gamma ray attenuation by EBL [6] is used to obtain the sensitivity curves for different redshifts. Also shown is the flux necessary for the observation of 1 photon above $10 \mathrm{GeV}$ by Fermi LAT.

A test array of seven tanks, called VAMOS (Verification And Measuring of Observatory System), was built on site in 2011. Six of the tanks were filled with water and instrumented with 4 to 7 PMTs per tank. Over 3 months of raw untriggered data have been collected with this array. Operation of the first 30 HAWC tanks is expected to start in 2012.

\section{First science with VAMOS}

The data collected with VAMOS can be used to search for high energy emission from GRBs, although with $\mathrm{a} \approx 20$-fold reduced sensitivity compared to the full HAWC array. Such an analysis has been performed for a long-duration, intense GRB 111016B detected by the IPN network (GCN circular 12452). The GRB occurred at a zenith angle of $32^{\circ}$ in the VAMOS field of view. No redshift information is available for this GRB. The number of events detected during a $155 \mathrm{~s}$ time interval around the GRB (including $5 \mathrm{~s}$ before T0 $=22: 41: 40$ UT plus the reported GRB duration) and reconstructed within $6^{\circ}$ from the GRB position was compared to the background estimate based on the event rate in the same angular bin during a $7 \mathrm{hr}$ period surrounding the GRB. A negative fluctuation of $\approx 0.6 \sigma$ was found. A $90 \%$ C.L. upper limit on the number of signal events was derived following the method of Feldman and Cousins [7]. The limit was then converted to flux units using a Monte Carlo simulation of the detector response. The limits computed for two different energy bands are presented in Fig. 2. Assuming a power law spectrum with a cutoff at $100 \mathrm{GeV}$ the upper limit on $\mathrm{E}^{2} \mathrm{dN} / \mathrm{dE}$ at $65 \mathrm{GeV}$ is $8.6 \cdot 10^{-4} \mathrm{erg} / \mathrm{cm}^{2}$. For a spectrum extending up to $316 \mathrm{GeV}$, the limit on the $>100 \mathrm{GeV}$ emission is $1.5 \cdot 10^{-4} \mathrm{erg} / \mathrm{cm}^{2}$ at $208 \mathrm{GeV}$. The analysis of GRB 111016B using the scalers technique will improve the limits. Note that, in similar conditions, the VAMOS sensitivity is comparable to the one of the much larger Milagro experiment that operated at a lower altitude of $2630 \mathrm{~m}$ (see e.g. [8]). 


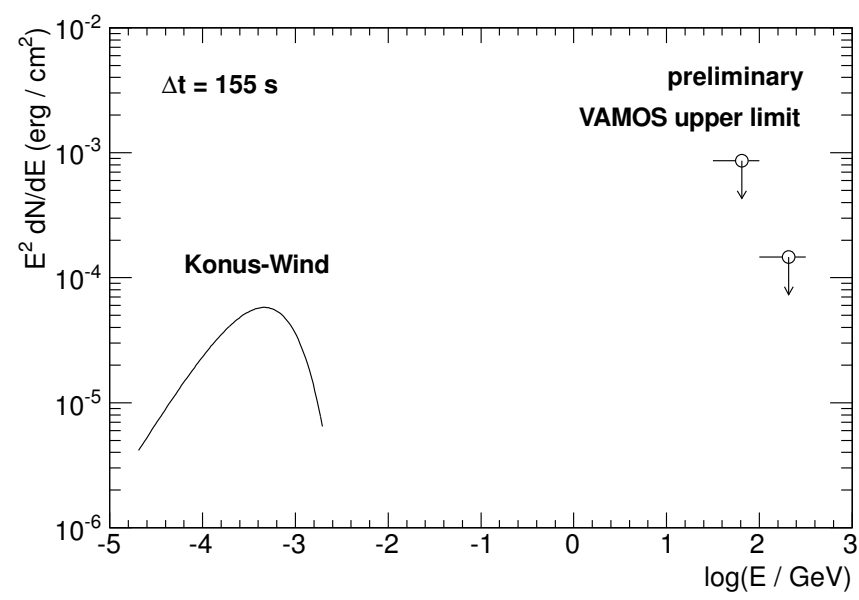

Figure 2: Upper limit on high energy emission from GRB 111016B imposed by VAMOS main DAQ data. The limit is given at $90 \%$ confidence level for two energy bands ( 31.6 to $100 \mathrm{GeV}$ and 100 to $316 \mathrm{GeV}$ ). The spectral fit reported by Konus-Wind (GCN circular 12456) is shown for comparison.

\section{Conclusion}

HAWC is a new generation wide field of view gamma-ray telescope currently under construction in Mexico. The high altitude, high duty cycle and large field of view make HAWC a suitable detector for gamma-ray bursts. HAWC will provide a realistic opportunity to observe the highenergy power law components of GRBs that extend at least up to $30 \mathrm{GeV}$. HAWC measurements will provide valuable information on the high-energy cutoff in the intrinsic GRB spectra and/or EBL absorption cutoff. An engineering array consisting of 6 water tanks has been operated at the HAWC site since September 2011. The collected data were used to set an upper limit on high energy emission from GRB 111016B.

\section{Acknowledgments}

The project is supported by the National Science Foundation, the US Department of Energy Office of High-Energy Physics, the LDRD program of Los Alamos National Laboratory and the University of Wisconsin Alumni Research Foundation, in U.S.; Consejo Nacional de Ciencia y Tecnología, Red de Física de Altas Energías, DGAPA-UNAM and VIEP-BUAP, in Mexico.

\section{References}

[1] A. Abdo et al., Fermi Observations of GRB 090902B: A Distinct Spectral Component in the Prompt and Delayed Emission, ApJ 706 (2009) L138

[2] M. Ackermann et al., Fermi Observations of GRB 090510: A Short Hard Gamma-Ray Burst with an Additional, Hard Power-Law Component from $10 \mathrm{keV}$ to GeV Energies, ApJ 716 (2010) 1178

[3] D. Band et al., BATSE observations of gamma-ray burst spectra. I - Spectral diversity, ApJ 413 (1993) 281

[4] R. Atkins et al., The High-Energy Gamma-Ray Fluence and Energy Spectrum of GRB 970417a from Observations with Milagrito, ApJ 583 (2003) 824 
[5] A. U. Abeysekara et al., On the sensitivity of the HAWC observatory to gamma-ray bursts, Astropart. Phys. 35 (2012) 641

[6] R. C. Gilmore et al., GeV gamma-ray attenuation and the high-redshift UV background, MNRAS 399 (2009) 1694

[7] G. J. Feldman, R. D. Cousins, Unified approach to the classical statistical analysis of small signals, Phys. Rev. D 57 (1998), 3873

[8] D. A. Williams et al., Search for GeV Emission from Gamma-Ray Bursts Using Milagro Scaler Data, AIP Conf. Proc. 921 (2007) 476 